\title{
CACTÁCEAS DO GÊNERO PERESKIA: COMPOSIÇÃO NUTRICIONAL E
} ALGUMAS APLICAÇÕES ${ }^{1}$

\author{
Lara Soares Santos ${ }^{2}$ \\ Carla Regina Amorim dos Anjos Queiroz \\ Cláudia Maria Tomás Melo
}

\begin{abstract}
RESUMO
O gênero Pereskia se destaca por teores de vários compostos nutricionais. No Brasil, Pereskia aculeata e Pereskia grandifolia são popularmente conhecidos como ora-pro-nóbis. As folhas de ambas são usadas como alimento em algumas regiões do Brasil, onde são encontradas também outras espécies, como a Pereskia bleo. Essas espécies têm apresentado importância nas áreas da agronomia, alimentos, medicina e pecuária. A importância das espécies motivou a realização de um estudo bibliográfico que contribuirá para a divulgação conjunta de resultados de pesquisa sobre essa planta, levando à divulgação do conhecimento científico e possivelmente também ao melhor aproveitamento dessas espécies como alimento humano. Muitos estudos têm sido publicados com os cactos do gênero Pereskia. A cada ano tem aumentado o número de pesquisas devido a sua importância. São plantas que apresentam altos teores de minerais, proteínas, fenóis em quantidades que as posicionam como a fonte alimentar desses nutrientes.
\end{abstract}

Palavras-chave: Composição química. Hortaliça não convencional. Ora-pro-nóbis. Planta alimentícia não convencional (PANC).

\section{CACTUS Pereskia: NUTRITIONAL COMPOSITION AND SOME APPLICATIONS}

\begin{abstract}
The genus Pereskia stands out for its content of several nutritional compounds. In Brazil, Pereskia aculeata and Pereskia grandifolia are popularly known as ora-pro-nóbis. The leaves of both plants are used as food in some regions of Brazil, where other species such as Pereskia bleo are also found. These species have shown importance in areas of agronomy, food, medicine and livestock. The importance of the species motivated the realization of a bibliographical study that will contribute to the joint dissemination of research results on this plant, leading to the dissemination of scientific knowledge and possibly also to the better use of these species as human food. Many studies have been published with the cacti of the genus Pereskia. Each year the quantity of researches has increased, due to its importance. They are plants that present high levels of minerals, proteins, phenols in quantities that position them as the food source of these nutrients.
\end{abstract}

\footnotetext{
${ }^{1}$ Como citar este artigo:

SANTOS, L. S.; QUEIROZ, C. R. A. A.; MELO, C. M. T. Cactáceas do gênero Pereskia: composição nutricional e algumas aplicações. ForScience, Formiga, v. 9, n. 2, e00702, jul./dez. 2021. DOI: 10.29069/forscience.2021v9n2.e702.

${ }^{2}$ Autor para correspondência: Lara Soares Santos, e-mail: lara.s3@hotmail.com.
} 
Keywords: Chemical composition. Non-conventional vegetable. Ora-pro-nóbis. Unconventional food plants.

\section{INTRODUÇÃO}

A diversidade vegetal brasileira é a maior do mundo, com mais de 56.000 espécies de plantas superiores, que correspondem a cerca de $19 \%$ de todas as espécies conhecidas, incluindo 162 espécies da família Cactaceae, das quais 123 são consideradas endêmicas do Brasil (GIULIETTI et al., 2005).

A Família Cactaceae é originária de regiões temperadas e tropicais, de clima quente e seco. São plantas suculentas, hábito variado e geralmente espinhosas. O gênero Pereskia é considerado o menos avançado da família, possuindo caule lenhoso bem desenvolvido, folhas suculentas e flores terminais (FARAGO et al., 2004).

As cactáceas do gênero Pereskia apresentam-se distribuídas em 17 espécies: P. aculeata Miller, P. aureiflora F. Ritter, P. bahiensis Gürke, P. bleo (Kunth) DC., P. diaz-romeroana Cárdenas, P. guamacho, P. grandifolia Haw., P. horrid DC., P. lychnidiflora DC., P. marcanoi Areces, P. nemorosa Rojas Acosta, P. portulacifolia (L.) DC., P. quisqueyana Alain, P. sacharosa Griseb., P. stenantha F. Ritter, P. weberiana K. Schum. e P. zinniiflora DC (PINTO; SCIO, 2014).

Entre as cactáceas encontradas no Brasil, o gênero Pereskia merece especial atenção. Considerada espécie nativa não endêmica (ZAPPI; MACHADO, 2010), a P. aculeata é conhecida e utilizada como alimento humano e configura como hortaliça não convencional em documentos oficiais brasileiros (BRASIL, 2004, 2006, 2010).

O gênero Pereskia se destaca por teores de vários compostos nutricionais. No Brasil, $P$. aculeata e P. grandifolia são popularmente conhecidos como ora-pro-nóbis. As folhas de ambas as plantas são usadas como alimento em algumas regiões do Brasil, onde são encontradas também outras espécies como a P. bleo (Figura 1) (PINTO; SCIO, 2014). 


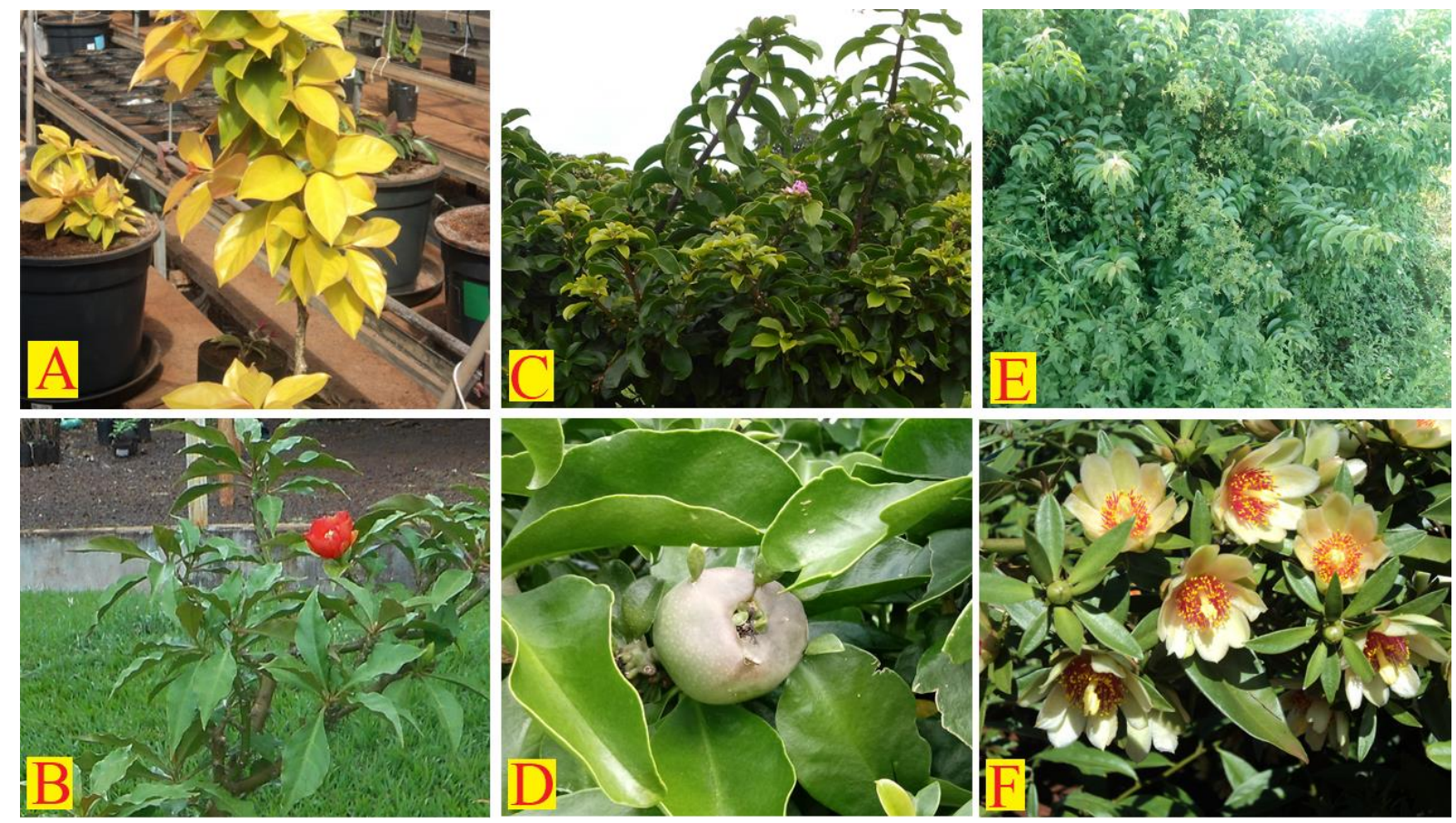

Figura 1 - Acessos de plantas do gênero Pereskia. A. Pereskia aculeata Godseffiana. B. Pereskia bleo com flores. C. Pereskia grandifolia Haw com flores. D. Frutos de Pereskia grandifolia. E. Pereskia aculeata Miller.

F. Flores de Pereskia aculeata Miller

Fonte: Autores (2014-2019).

Diversas espécies sub exploradas da flora brasileira podem ser fontes naturais de compostos bioativos, vitaminas, minerais e macronutrientes para complementação da alimentação humana e até mesmo animal. Nesse contexto situa-se a $P$. aculeata que é classificada como hortaliça folhosa não convencional, de sabor agradável, fácil preparo e considerada também, popularmente, como planta medicinal, em virtude das ações antiinflamatórias, analgésicas e dermatológicas (KINUPP; BARROS, 2008; PINTO; SCIO, 2014).

Um problema ao explorar as folhas de vegetais não convencionais é a falta de caracterização da planta e a possibilidade de existência de substâncias com caráter antinutricional, também chamados fatores antinutricionais, que podem reduzir e interferir na biodisponibilidade e digestibilidade de alguns nutrientes. Santos (2006) e Silveira (2015) realizaram estudos para a caracterização de compostos com essas características em P. aculeata.

Dias et al. (2005) afirmaram que há pouco conhecimento de suas propriedades e pouca divulgação das plantas do gênero Pereskia justificando assim o baixo consumo pela população. Porém, os estudos vêm aumentando a cada ano, afirmando cada vez mais a qualidade nutricional e utilização desse vegetal na alimentação humana (MORAES et al., 2018).

As cactáceas do gênero Pereskia têm se tornado mais importantes em várias áreas do conhecimento, tais como agronomia, alimentos, medicina e pecuária. Sua utilização in natura ou previamente seca em produtos alimentícios, como doces e salgados, na medicina em 
tratamentos anticâncer, em pomadas anti-inflamatórias, encapsulada, no combate à desnutrição pelo seu conteúdo em ferro, como chás e folhas secas para complementação nutricional, aplicada como antifúngico, antibacteriano, usada na pecuária adicionadas em rações e como emulsificante na indústria (ALMEIDA; CORREIA, 2012; SHARIF et al., 2013).

Motivados pela importância do aproveitamento de vegetais não convencionais como alimento e fonte de nutrientes, bem como pelo crescente número de estudos científicos e popularização por meio de canais digitais de comunicação, realizou-se um levantamento bibliográfico de trabalhos científicos sobre cactáceas do gênero Pereskia no período entre setembro de 2018 a maio de 2019. Foram utilizadas as fontes eletrônicas Google Acadêmico, Scielo e os acervos bibliográficos de revistas disponíveis online, com base em um conjunto de palavras-chave (Pereskia, composição química, Pereskia bleo, Pereskia aculeata, Pereskia aculeata Miller, Pereskia grandifolia, elaboração Pereskia, fármacos Pereskia) que permitisse chegar às informações sobre suas propriedades nutricionais como alimento, composição química, importância para alimentação e aplicação em alimentos e fármacos.

Este estudo contribuirá para a divulgação conjunta de resultados de pesquisa sobre essa planta, levando ao conhecimento e possivelmente também para o melhor aproveitamento dessas espécies como alimento humano.

\section{DESENVOLVIMENTO}

\subsection{Composição química}

Os primeiros estudos para avaliação da composição química de macronutrientes da folha da ora-pro-nóbis foram realizados na década de 70 por Almeida Filho e Cambraia (1974), que realizaram estudos com a espécie $P$. aculeata. Em 1977, 1991 e 2004 foram registrados outros estudos, porém somente a partir de 2009 houve maior interesse da comunidade científica para o estudo da espécie com maior número de publicações em 2015 (Tabela 1). 
Tabela 1 - Composição de folhas de cactáceas do gênero Pereskia, de acordo com a literatura. Para os parâmetros proteína, cinzas, fibras e lipídeos

\begin{tabular}{|c|c|c|c|c|c|}
\hline Espécie & $\begin{array}{l}\text { Proteína } \\
\text { g } 100 \mathrm{~g}^{-1^{3}}\end{array}$ & $\begin{array}{l}\text { Cinzas } \\
\text { g } 100 \mathrm{~g}^{-1}\end{array}$ & $\begin{array}{c}\text { Fibras } \\
\text { g } 100 \mathrm{~g}^{-1}\end{array}$ & $\begin{array}{l}\text { Lipídeos } \\
\text { g } 100 \mathrm{~g}^{-1}\end{array}$ & Referência \\
\hline \multirow{2}{*}{$\begin{array}{l}\text { Pereskia } \\
\text { aculeata } \\
\text { Godseffiana }\end{array}$} & $13,4 \pm 3,60$ & $26,3 \pm 1,80$ & $13,5 \pm 0,20$ & $3,6 \pm 0,60$ & SANTOS et al. (2015) \\
\hline & $18,2 \pm 0,30$ & $28,33 \pm 3,41$ & $16,69 \pm 2,80$ & $2,9 \pm 0,23$ & GUIMARÃES (2018) \\
\hline \multirow{3}{*}{$\begin{array}{l}\text { Pereskia } \\
\text { aculeata }\end{array}$} & 25,0 & - & - & - & $\begin{array}{l}\text { KINUPP; BARROS } \\
\text { (2008) }\end{array}$ \\
\hline & $29,0 \pm 0,59$ & $14,8 \pm 0,18$ & $21,6 \pm 0,82$ & $5,1 \pm 0,15$ & $\begin{array}{l}\text { ALMEIDA et al. } \\
\text { (2014) }\end{array}$ \\
\hline & 23,4 & - & - & - & SOUZA et al. (2016) \\
\hline \multirow{13}{*}{$\begin{array}{l}\text { Pereskia } \\
\text { aculeata } \\
\text { Miller }\end{array}$} & 25,5 & 20,1 & 9,1 & 6,8 & $\begin{array}{l}\text { ALMEIDA FILHO; } \\
\text { CAMBRAIA (1974) }\end{array}$ \\
\hline & 25,4 & 19,2 & 7,1 & 5,8 & $\begin{array}{l}\text { DAYRELL; VIEIRA } \\
\text { (1977) }\end{array}$ \\
\hline & & & & & ALBUQUERQUE; \\
\hline & 28,6 & 14,2 & 7,7 & 6,3 & SABAA-SRUR; \\
\hline & & & & & FREIMAN (1991) \\
\hline & 19,67 & - & 29,6 & 4,4 & GIRÃO et al. (2003) \\
\hline & $28,4 \pm 0,40$ & $16,1 \pm 0,10$ & $9,8 \pm 0,20$ & $4,1 \pm 0,3$ & TAKEITI et al. (2009) \\
\hline & $22,9 \pm 3,02$ & $18,1 \pm 0,06$ & $12,6 \pm 1,38$ & $3,6 \pm 0,41$ & ALMEIDA (2012) \\
\hline & 27,8 & 20,1 & - & - & $\begin{array}{l}\text { GONÇALVES et al. } \\
\text { (2014) }\end{array}$ \\
\hline & 18,9 & 17,5 & 31,4 & 4,01 & $\begin{array}{l}\text { RODRIGUES et al. } \\
\text { (2014) }\end{array}$ \\
\hline & 26,5 & 32,5 & 44,7 & 5,7 & QUEIROZ et al. (2015) \\
\hline & $23,3 \pm 0,20$ & $20,6 \pm 0,49$ & $51,0 \pm 0,56$ & $1,4 \pm 0,10$ & SILVEIRA (2015) \\
\hline & $17,9 \pm 0,75$ & $22,8 \pm 0,27$ & - & $3,2 \pm 0,20$ & VARGAS (2017) \\
\hline \multirow{5}{*}{ Pereskia bleo } & & & & & ALBUQUERQUE; \\
\hline & 17,38 & 16,24 & 7,38 & 7,48 & SABBA-SRUR; \\
\hline & & & & & FREIMAN (1991) \\
\hline & $12,9 \pm 1,6$ & $24,9 \pm 3,1$ & $16,2 \pm 5,4$ & $4,2 \pm 0,5$ & SANTOS et al. (2015) \\
\hline & $18,6 \pm 0,59$ & $26,2 \pm 1,34$ & $1,6 \pm 0,22$ & $55,7 \pm 8,92$ & GUIMARÃES (2018) \\
\hline \multirow{3}{*}{$\begin{array}{l}\text { Pereskia } \\
\text { grandifolia }\end{array}$} & $32,0 \pm 0,46$ & $12,6 \pm 0,06$ & $18,8 \pm 0,92$ & $6,7 \pm 0,30$ & $\begin{array}{l}\text { ALMEIDA et al. } \\
\text { (2014) }\end{array}$ \\
\hline & $15,9 \pm 0,80$ & $27,0 \pm 3,70$ & $16,4 \pm 2,90$ & $6,3 \pm 1,30$ & SANTOS et al. (2015) \\
\hline & $27,7 \pm 0,60$ & $16,1 \pm 0,23$ & $52,8 \pm 0,50$ & $2,2 \pm 0,01$ & SILVEIRA (2015) \\
\hline
\end{tabular}

${ }^{3} \mathrm{O}$ mesmo que percentual $\%$. 


\begin{tabular}{|c|c|c|c|c|c|}
\hline & $24,2 \pm 0,75$ & $29,6 \pm 4,26$ & $5,5 \pm 0,93$ & $0,4 \pm 0,10$ & GUIMARÃES (2018) \\
\hline Média & $23,8 \pm 5,40$ & $20,1 \pm 5,80$ & $16,3 \pm 15,70$ & $4,4 \pm 11,90$ & - \\
\hline
\end{tabular}

Para as espécies P. aculeata Godseffiana e P. bleo foram encontrados menores números de estudos científicos. A $P$. aculeata Miller é a espécie de maior alvo de estudos, com 11 pesquisas descritas nessa revisão (Tabela 1).

\subsubsection{Proteínas}

As proteínas são encontradas em alimentos de origem animal em percentuais elevados. Porém, pessoas de baixo poder aquisitivo, podem ter acesso limitado a essas fontes de proteínas, o que faz das hortaliças não convencionais, baratas e abundantes, uma alternativa de consumo desses nutrientes e uma opção para pessoas com hábitos alimentares diferenciados (KINUPP; BARROS, 2008).

A P. aculeata é conhecida popularmente em algumas regiões como "carne de pobre" pelo seu alto teor de proteína. Santos et al. (2015) estudaram o teor de proteínas em três diferentes espécies (Tabela 1) e verificaram que os teores são iguais entre si. Verificações experimentais mostraram que as espécies de P. aculeata Miller, P. aculeata Godseffiana e $P$. bleo, cozidas, liofilizadas ou in natura, apresentam o mesmo conteúdo proteico.

As plantas analisadas por Guimarães (2018) foram conduzidas em campo experimental com irrigação por gotejamento 2 vezes ao dia, no período seco e, com 30 minutos de irrigação no período chuvoso, sendo realizado um estudo nas mesmas espécies que Santos et al. (2015) e obteve resultado maior para o teor de proteína. As amostras de Santos et al. (2015) foram cultivadas em ambiente com teto coberto por filme plástico. O único parâmetro encontrado por Guimarães (2018) inferior ao estudo de Santos et al. (2015) foi o teor de fibras nas espécies $P$. bleo e $P$. grandfolia sendo na espécie $P$. bleo o menor teor de fibra encontrado em todas as espécies deste estudo.

O maior teor de proteína foi nas espécies analisadas por Almeida et al. (2014) ( $P$. aculeata com $28,99 \% \pm 0,59 \%$ e P. grandfolia $32,02 \% \pm 0,46 \%$ ), com plantas colhidas em residências da cidade de São Gonçalo do Abaeté/MG, no mês de outubro de 2010; porém, esse mesmo pesquisador obteve os menores teores de cinzas nas espécies $P$. aculeata e $P$. grandfolia.

A média do teor de proteínas encontradas nos estudos apresentados foi de 23,8\% $\pm 5,40 \%$ (Tabela 1), considerando 23 estudos. O teor de proteínas é influenciado pela interação entre as 
lâminas de irrigação aplicadas e as doses de composto orgânico no substrato. O aumento nas doses do composto tem mais influência do que o aumento nas lâminas de irrigação. O teor de proteína na folha aumenta com o aumento da dose do composto orgânico no substrato (ANDRADE, 2012).

O teor de proteína apresentado na Tabela 1 demonstra que pode variar entre 13,4\% \pm $3,6 \%$ para a $P$. aculeata Godseffiana a 32,02\% $\pm 0,46 \%$ para $P$. grandfolia. Além da proteína ser influenciada pelos fatores irrigação e substrato, algumas técnicas de manejo também podem influenciar no teor de proteína das folhas de Pereskia. O sombreamento nas plantas é um fator de interferência no teor de proteína. O maior teor de proteínas nas folhas é encontrado em condição totalmente sombreada (QUEIROZ et al., 2015).

Vargas (2017) avaliou as folhas de P. aculeata quando coletadas no verão e no inverno e foram encontrados os maiores teores de proteínas, carboidratos e lipídios quando colhidas no verão.

Entre os 20 aminoácidos formadores de proteínas, 10 são conhecidos como essenciais. Os aminoácidos essenciais são aqueles que devem ser incluídos na dieta e que não são sintetizados pelo nosso organismo (PINHEIRO; PORTO; MENEZES, 2005). Alguns desses aminoácidos podem ser encontrados no gênero Pereskia como a lisina, histidina, arginina, treonina, valina, metionina, isoleucina, leucina e fenilalanina (DAYRELL; VIEIRA, 1977; ALBUQUERQUE; SRUR; FREIMAN, 1991; TAKEITI et al., 2009).

A digestibilidade da proteína também é alvo de estudos. Em amostras de P. aculeata avaliadas por Takeiti et al. (2009) a digestibilidade foi de 75,9\%, abaixo dos valores encontrados por Almeida Filho e Cambraia (1974) para a mesma espécie, 90,2\% de digestibilidade proteica em folhas colhidas em Viçosa/ MG e 79,5\% de digestibilidade em folhas colhidas em Guiricema/MG. Dayrell e Vieira (1977) obtiverem valores 78,86\% de digestibilidade proteica, também nas amostras analisadas de folhas de P. aculeata Miller.

\subsubsection{Minerais}

Quanto aos elementos minerais, também chamados de cinzas ou resíduo mineral, sabese que são largamente distribuídos na natureza e exercem importantes funções no organismo humano. O corpo humano, em condições normais, excreta diariamente de 20 a $30 \mathrm{~g}$ de minerais e necessita de reposição imediata por meio da alimentação (FRANCO, 2004). 
Segundo Del-Vechio et al. (2005), o uso de concentrados de minerais e vitaminas pode ser feito por meio de farelos ou pó de folhas ou de sementes, como uma forma de fornecimento de nutrientes que são indispensáveis para manter a saúde, desde que adicionados em doses mínimas à alimentação tradicional, constantemente.

A $P$. aculeata, devido às suas características e propriedades, representa uma alternativa para enriquecimento e incremento da qualidade da alimentação, pois suas folhas possuem importantes qualidades nutritivas, como alto teor de cálcio, fósforo, magnésio, ferro e cobre (ALMEIDA FILHO; CAMBRAIA, 1974; MERCÊ et al., 2001).

O teor de minerais apresentado pelos resultados de diversas pesquisas (Tabela 1), comprova a qualidade nutricional das plantas do gênero Pereskia. Quando comparado o teor de minerais em vegetais folhosas convencionais, essas plantas apresentam-se com teores bem maiores, podendo ser verificados até o dobro de cada nutriente. Na Tabela Brasileira de Composição de Alimentos (TACO), entre as hortaliças folhosas cruas convencionais com maiores teores de minerais, encontram-se, em base seca, 14,3\% (agrião), 15,9\% (almeirão e salsa) e 21,2\% (rúcula) (NEPA, 2009). Nas plantas do gênero Pereskia pode-se obter até 29,57\% de cinzas, valor expressivo frente às hortaliças convencionais (Tabela 1).

A $P$. grandfolia apresentou maior teor de minerais $29,57 \% \pm 4,26 \%$ em estudo realizado por Guimarães (2018) já a $P$. aculeata obteve o menor teor $14,81 \% \pm 0,18 \%$ em estudo feito por Almeida et al. (2014) (Tabela 1).

Segundo estudo de Takeiti et al. (2009), considerando os microelementos, as folhas da P. aculeata são fontes ricas em manganês (46,4 mg $\left.100 \mathrm{~g}^{-1}\right)$, zinco $\left(26,71 \mathrm{mg} 100 \mathrm{~g}^{-1}\right)$ e ferro

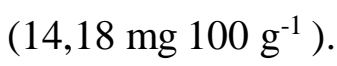

\subsubsection{Fibra Bruta}

As plantas estudadas por Silveira (2015) foram colhidas no Horto de Plantas Medicinais no Departamento de Agricultura da Universidade Federal de Lavras (UFLA) e, o teor de fibras nas espécies analisadas foram os maiores apresentados nesse estudo. A metodologia utilizada, diferentemente dos demais autores, foi pelo processo de espectrofotometria.

A $P$. aculeata e $P$. grandifolia apresentaram as maiores quantidades de fibras com 51,03 $\pm 0,56 \%$ e $52,83 \% \pm 0,50 \%$, respectivamente (SILVEIRA, 2015). A P. bleo estudada por Guimarães (2018) apresentou o menor teor de fibras com 1,58\% $\pm 0,22 \%$. 


\subsubsection{Lipídeos}

Um aspecto positivo das plantas do gênero Pereskia é a baixa quantidade de lipídios, podendo ser utilizadas em dietas hipocalóricas e com restrição de lipídeos (RODRIGUES et al., 2014). O teor de lipídeos com menor concentração foi observado por Guimarães (2018) com $0,42 \% \pm 0,10 \%$ em $P$. grandifolia, porém o maior teor foi avaliado também por ele com quantidade bastante elevada para vegetais folhosos sendo encontrado $55,71 \% \pm 8,92 \%$ na $P$. bleo.

O teor de lipídeos em $P$. aculeata pode variar em função da oferta de água no solo ou no substrato, desde que as demais variáveis edáficas, nutricionais e ambientais sejam controladas, possibilitando manejo adequado aos interesses de maximização de determinados constituintes químicos na planta (QUEIROZ et al., 2015). Vargas (2017) afirma que, a fração lipídica não é influenciada pela sazonalidade.

Girão et al. (2003), Takeiti et al. (2009) e Rodrigues et al. (2014), avaliaram teores de lipídeos que foram próximos 4,41\%, 4,1\% e 4,01\%, nas folhas de $P$. aculeata, respectivamente. Já Almeida Filho e Cambraia (1974), Dayrell e Vieira (1977), Albuquerque, Sabaa-Srur e Freiman (1991), Queiroz et al. (2015) encontraram 6,8\%, 5,83\%, 6,30\% e 5,7\%, respectivamente (Tabela 1$)$.

Assim, a baixa quantidade de lipídios de $P$. aculeata e de $P$. grandfolia torna estas hortaliças uma fonte alimentar importante para indivíduos que necessitam de dietas hipolipídicas (RODRIGUES et al., 2014).

\subsubsection{Fenóis}

Os fenóis são substâncias com presença de anel aromático e um ou mais grupos hidroxílicos e possuem estrutura variável, podendo ser considerados multifuncionais. Essas substâncias são estudadas como potencial fonte antioxidante e estão presentes naturalmente em vegetais (ANGELO; JORGE, 2007). Vários estudos sugerem o consumo de plantas que contenham compostos fenólicos, pois podem contribuir significativamente para saúde humana (MELO et al., 2006; CERQUEIRA; GENNARI; AUGUSTO, 2007; SILVA et al., 2010). Santos et al. (2015) estudaram o conteúdo de compostos fenólicos em folhas de Pereskia e, mostraram a presença de até 159,5 $\pm 56,5 \mathrm{mg}$ EAG (equivalentes de ácido gálico) $\mathrm{g}^{-1}$ folha seca. Estes valores estão próximos ou são maiores que outros vegetais medicinais, indicando que as 
cactáceas do gênero Pereskia podem apresentar propriedades benéficas quando consumidas. De acordo com Almeida et al. (2014) a quantidade de compostos fenólicos encontrado nas folhas de $P$. aculeata não são prejudiciais à digestibilidade das proteínas.

Mandelli (2016) demonstrou que os frutos da ora-pro-nóbis podem serem ricos em compostos fenólicos, apresentando teores de $392 \pm 0,083 \mathrm{mg}$ EAG/100 g de fruto, elevado potencial de remoção dos radicais DPPH $\left(820,06 \mathrm{mM} \mathrm{g}^{-1}\right)$. Os resultados sugerem que os frutos podem ser empregados na alimentação como fonte de antioxidantes, podendo ser consumidos in natura, ou sendo incorporado em outros alimentos.

De acordo com Souza (2014), há atividade antioxidante em extratos frescos, refrigerados e congelados de folhas de $P$. aculeata, obtidos com infusão em água e extração em etanol, metanol e água.

\subsection{Utilização em alimentos}

A utilização de folhas de Pereskia em alimentos, processados ou não, já vem sendo estudada como forma de enriquecer nutricionalmente e principalmente associada ao tratamento da anemia ferropriva, câncer, osteoporose e à constipação intestinal (ALMEIDA; CORREIA, 2012).

Rocha et al. (2008) adicionaram folhas secas de Pereskia à massa de macarrão tipo talharim e os maiores teores de proteínas, fibras e cinzas foram observados na massa adicionada de folhas de Pereskia do que em macarrão convencional, em função da adição de 2,0\% desta hortaliça previamente desidratada.

O estudo da ação antioxidante de Pereskia aplicada em mortadela comprovou seu potencial na indústria cárnea de embutidos (RODRIGUES, 2016). Também foi realizado estudo para verificar a ação emulsificante em mortadela, reforçando que a Pereskia surge como importante estratégia para obtenção de derivados com qualidade nos atributos de textura, bem como na redução do teor de lipídeos e valor calórico (LISE, 2018).

Estudos com adição de folhas de Pereskia em pão foram realizados e verificou-se que a Pereskia é viável na preparação de pães, os quais possuem bom índice de aceitação global (MARTINEVSKI et al., 2013).

Outros estudos adicionando Pereskia na elaboração de produtos como bolos, picolé, cupcakes, farinha, como suplementos alimentícios também foram avaliados em sua aceitação 
e/ou composição nutricional (SILVA et al., 2014; MARINELLI, 2016; PAULA et al., 2016; SANTANA et al., 2018).

\subsection{Aplicação em fármacos}

O gênero Pereskia tem sido estudado também em fármacos, sendo sua utilização evidenciada em produtos cosméticos, de higiene pessoal e até como medicamentos fitoterápicos (SANTOS et al., 2011).

Estudos realizados em $P$. bleo em utilização como medicamento, mostraram inibição notável contra células cancerosas. Compostos isolados a partir de P. bleo e P. grandfolia também mostraram atividade anticâncer. A sua não toxicidade contra células normais é uma grande vantagem na utilização das folhas. O potencial desses compostos deve ser explorado por causa da procura de produtos naturais para tratamento de doenças (SHARIF et al., 2013).

Barros et al. (2010) desenvolveram uma pomada contendo extrato de P. aculeata e verificaram que este produto apresentou resultados satisfatórios quanto à análise macro e microscópica das feridas, sugerindo que a mesma proporcionou uma melhora no processo de cicatrização.

A ação antioxidante, ao comparar com cosméticos anti-idade e o creme de $P$. aculeata, apresentou atividade antioxidante próximo a 75\%, estando próximo das amostras comerciais analisadas, podendo, dessa forma, o extrato ser utilizado como ativo antioxidante em formulações cosméticas para prevenção do envelhecimento cutâneo (SOUZA; SATOR; FELIPE, 2013).

Santos et al. (2010) avaliaram o perfil de susceptibilidade do extrato bruto das folhas de P. aculeata sobre patógenos bucal Enterococcus faecalis (ATCC 4082), Streptococcus mutans (ATCC 25175), Lactobacillus casei (ATCC 11578) e Candida albicans (ATCC 18804 e 448858) e, observaram que houve uma inibição no crescimento de E. faecalis, quando exposto à ação do extrato de $P$. aculeata.

Garcia et al. (2011) avaliaram a ação antioxidante dos extratos de própolis verde e vermelha e da $P$. aculeata e afirmaram que ela tem melhor ação antioxidante do que as amostras de própolis testadas. A partir da utilização padronizada desses extratos em produtos cosméticos, os autores indicam a utilização de extratos de $P$. aculeata em formulações cosméticas destinadas a prevenção do envelhecimento cutâneo. 


\section{CONSIDERAÇÕES FINAIS}

As plantas do gênero Pereskia tiveram aumento nos estudos investigativos nos últimos anos, e atualmente é material vegetal de grande interesse para pesquisadores. As avaliações têm investigado seus compostos químicos, formas de aproveitamento das partes das plantas para produção de alimentos e para desenvolvimentos de produtos fármacos. São consideradas fontes de compostos bioativos.

O gênero possui um potencial para ser usado como complemento nutricional na alimentação, utilização como emulsificante na indústria, como bactericida, e agente antioxidante e possui ação anticâncer.

\section{REFERÊNCIAS}

ALBUQUERQUE, M. G. P. T; SABAA-SRUR, A. U. O.; FREIMAN, L. O. Composição centesimal e escore de amino-ácidos em três espécies de 'ora-pro-nobis' (Pereskia aculeata Mill., P. bleu De Candolle e P. pereskia (L) Karsten). Boletim SBCTA, Campinas, v. 25, n. 1, p. 7-12, 1991.

ALMEIDA, M. E. F de. Farinha de folhas de cactáceas do gênero Pereskia: caracterização nutricional e efeito sobre ratos wistar submetidos à dieta hipercalórica. 2012. 126 f. Tese (Doutorado em Agronomia) - Universidade Federal de Lavras, Lavras, 2012. Disponível em: http://repositorio.ufla.br/jspui/handle/1/585. Acesso em: 14 set. 2018.

ALMEIDA, M. E. F.; CORRÊA, A. D. Utilização de cactáceas do gênero Pereskia na alimentação humana em um município de Minas Gerais. Ciência Rural, Santa Maria, v. 42, n. 4, p. 751-756, 2012. Disponível em: https://www.scielo.br/pdf/cr/v42n4/a11112cr5075.pdf. Acesso em: $1^{\circ}$ set. 2018.

ALMEIDA, M. E. F. et al. Caracterização química das hortaliças não-convencionais conhecidas como ora-pro-nobis. Bioscience Journal, Uberlândia, v. 30, n. 1, p. 431-439, 2014. Disponível em:

http://www.seer.ufu.br/index.php/biosciencejournal/article/view/17555/14557. Acesso em: 12 set. 2018.

ALMEIDA-FILHO, J.; CAMBRAIA, J. Estudo do valor nutritivo do "ora-pro-nóbis" (Pereskia aculeata Mill.). Revista Ceres, Viçosa, v. 21, n. 114, p. 105-111, 1974.

ANDRADE, R. R. Substrato e irrigação em ora-pro-nóbis (Pereskia aculeata Mill.). 2012. 90 f. Tese (Doutorado em Ciências Agrárias e Veterinárias) - Universidade Estadual Paulista "Júlio de Mesquita Filho", Jaboticabal, 2012. Disponível em: https://repositorio.unesp.br/bitstream/handle/11449/100843/andrade_rr_dr_jabo.pdf?sequence =1\&isAllowed=y. Acesso em: 12 nov. 2018. 
ANGELO, P. M.; JORGE, N. Compostos fenólicos em alimentos: uma breve revisão. Revista Instituto Adolfo Lutz, São Paulo, v. 66, n. 1, p. 232-240, 2007. Disponível em:

http://periodicos.ses.sp.bvs.br/pdf/rial/v66n1/v66n1a01.pdf. Acesso em: 10 set. 2018.

BARROS, K. N. et al. Desenvolvimento de formulação de uso tópico com ação cicatrizante contendo extrato de Pereskia aculeata. Revista Iniciação Científica Cesumar, Maringá, v. 12, n. 1, p. 29-37, 2010. Disponível em:

https://periodicos.unicesumar.edu.br/index.php/iccesumar/article/view/1250/1059. Acesso em: 10 dez. 2018.

BRASIL. Guia alimentar para a população brasileira. Ministério da Saúde. Brasília, 2004, 120 p. Disponível em:

https://bvsms.saude.gov.br/bvs/publicacoes/guia_alimentar_populacao_brasileira_2ed.pdf. Acesso em: 10 set. 2018.

BRASIL. Decreto 5.813, de 22 de junho de 2006. Aprova a Política Nacional de Plantas Medicinais e Fitoterápicos e dá outras providências. Presidência da República. Brasília, DF, 2006. 60 p. Disponível em: https://www2.camara.leg.br/legin/fed/decret/2006/decreto5813-22-junho-2006-543661-publicacaooriginal-54192-pe.html. Acesso em: 12 ago. 2018.

BRASIL. Manual de hortaliças não-convencionais. Ministério da Agricultura, Pecuária e Abastecimento. Belo Horizonte, 2010. 92 p. Disponível em:

http://www.abcsem.com.br/docs/manual_hortalicas_web.pdf. Acesso em: 12 set. 2018.

CERQUEIRA, F. M.; MEDEIROS, M. H. G. de; AUGUSTO, O. Antioxidantes dietéticos: controvérsias e perspectivas. Química Nova, São Paulo, v. 30, n. 2, p. 441-449, 2007. Disponível em: http://www.scielo.br/pdf/qn/v30n2/35.pdf. Acesso em: 11 set. 2018.

DAYRELL, M. S.; VIEIRA, E. C. Leaf protein concentrate of the cactacea Pereskia aculeata Mill. Extraction and composition. Nutrition Reports International, Stoneham, Massachusetts, v. 15, n. 5, p. 529-537, 1977.

DEL-VECHIO, G. et al. Efeito no tratamento térmico em sementes de abóboras (Curcubita spp.) sobre os níveis de fatores antinutricionais e/ou tóxicos. Ciência e Agrotecnologia, Lavras, v. 29, n. 2, p. 369-376, 2005. Disponível em: http://www.scielo.br/pdf/cagro/v29n2/a14.pdf. Acesso em: 12 set. 2018.

DIAS, A. C. P. et al. Avaliação do consumo de hortaliças não convencionais pelos usuários das unidades do Programa Saúde da Família (PSF) de Diamantina-MG. Alimentos e Nutrição, Araraquara, v. 16, n. 3, p. 279-284, 2005 Disponível em: http://servbib.fcfar.unesp.br/seer/index.php/alimentos/article/viewArticle/481. Acesso em: 12 set. 2018.

FARAGO, P. V. et al. Análise morfoanatômica de folhas de Pereskia grandifolia Haw., Cactaceae. Acta Farmaceutica Bonaerense, Buenos Aires, Argentina, v. 23, n. 3, p. 323327, 2004. Disponível em:

http://www.latamjpharm.org/trabajos/23/3/LAJOP $23 \quad 3 \quad 1 \quad 8$ 7G0EKC07R0.pdf. Acesso em: 12 set. 2018.

FRANCO, G. Tabela de composição química dos alimentos. 9. ed. São Paulo: Atheneu, 2004. 
GARCIA, B. H. et al. Estudo da atividade antioxidante dos extratos de própolis e Pereskia aculeata. In: ENCONTRO INTERNACIONAL DE PARODUÇÃO CIENTÍFICA, 7., 2011, Maringá. Anais eletrônicos[...]. Maringá: Cesumar, 2011. Disponível em http://www.apiariosilvestre.com.br/images/documentos/4.Atidadeantioxidante.pdf. Acesso em: 19 jul. 2019.

GIULIETTI, A. M. et al. Biodiversidade e conservação das plantas no Brasil. Megadiversidade, Belo Horizonte, v. 1, n. 1, p. 52-61, 2005. Disponível em: http://www.agencia.cnptia.embrapa.br/Repositorio/BIOD ConservacaoIDeWNPNpKEJw.pdf. Acesso em: 12 nov. 2018.

GIRÃO, L. V. C. et al. Avaliação da composição bromatológica de ora-pro-nóbis. Horticultura Brasileira, Brasília, v. 21, n. 2, suplemento 1, p. 411, 2003. Disponível em: http://www.abhorticultura.com.br/biblioteca/arquivos/Download/Biblioteca/pmfi5000c.pdf. Acesso em: 12 ago. 2018.

GONÇALVES, J. P. Z. et al. Quantificação de proteínas e análise de cinzas encontradas nas folhas e caule da ora-pro-nobis (Pereskia aculeata Miller). In: CONGRESSO BRASILEIRO DE ENGRNHARIA QUÍMICA, 20., 2014, Florianópolis. Anais eletrônicos[...]. Florianópolis, 2014. Disponível em: http://pdf.blucher.com.br.s3-sa-east1.amazonaws.com/chemicalengineeringproceedings/cobeq2014/0167-26714-164573.pdf. Acesso em: 19 jul. 2019.

GUIMARÃES, J. R. A. Caracterização físico-química e composição minieral de Pereskia aculeata Mill., Pereskia grandifolia Haw. e Pereskia bleo (Kunth) DC. 2018. 72 f. Tese (Doutorado em Ciências Agronômicas) - Universidade Estadual de Paulista, Botucatu, 2018. Disponível em:

https://repositorio.unesp.br/bitstream/handle/11449/154805/guimaraes jra dr botfca.pdf?seq uence=3\&isAllowed=y. Acesso em: 1 jun. 2019.

KINUPP, V. F.; BARROS, I. B. I. de. Teores de proteína e minerais de espécies nativas, potenciais hortaliças e frutas. Ciência e Tecnologia de Alimentos, Campinas, v. 28, n. 4, p. 846 - 857, 2008. Disponível em: http://www.scielo.br/pdf/cta/v28n4/a13v28n4.pdf. Acesso em: 21 nov. 2018.

LISE, C. C. Potencial emulsificante de ora pro nóbis (Pereskia aculeata Miller) em derivado cárneo tipo mortadela. 2018. 48 f. Trabalho de Conclusão de Curso (Curso de Bacharelado em Química) - Universidade Tecnológica Federal do Paraná, Pato Branco, 2018. Disponível em:

http://repositorio.roca.utfpr.edu.br/jspui/bitstream/1/10893/1/PB_DAQUI_2018_1_2.pdf.

Acesso em: 5 maio 2019.

MANDELLI, M. K. de L. M. Avaliação dos parâmetros nutricionais e potencial antioxidante do fruto de ora-pro-nobis (Pereskia aculeata Miller). 2016. $34 \mathrm{f}$. Trabalho de Conclusão de Curso (Curso de Bacharelado em Química) - Universidade Tecnológica Federal do Paraná, Pato Branco, 2016. Disponível em: http://repositorio.roca.utfpr.edu.br/jspui/bitstream/1/6985/1/PB_DAQUI_2016_2_4.pdf. Acesso em: 12 abr. 2019. 
MARTINEVSKI, C. S. et al. Utilização de bertalha (Anredera cordifolia (Ten.) Steenis) e ora-pro-nobis (Pereskia aculeata Mill.) na elaboração de pães. Alimentos e Nutrição, Araraquara, v. 24, n. 3, p. 272, 2013. Disponível em: http://serv-

bib.fcfar.unesp.br/seer/index.php/alimentos/article/view/267/2251. Acesso em: 21 out. 018.

MELO, E. A. et al. Capacidade antioxidante de hortaliças usualmente consumidas. Ciência Tecnologia de Alimentos, Campinas, v. 26, n. 3, p. 639-644, 2006. Disponível em: http://www.scielo.br/pdf/cta/v26n3/31768.pdf. Acesso em: 15 set. 2018.

MERCÊ, A. L. et al. Complexes of arabinogalactan of Pereskia aculeata and $\mathrm{Co}^{2+}, \mathrm{Cu}^{2+}$, $\mathrm{Mn}^{2+}$, and $\mathrm{Ni}^{2+}$. Bioresource Technology, Amsterdã, Netherlands, v. 76, n.1, p.29-37, 2001. Disponível em: http://europepmc.org/article/med/11315807. Acesso em: 12 set. 2018.

MORAES, T. V. de et al. Composição química da espécie Pereskia aculeata Miller: uma análise bibliométrica. Brazilian Journal of Surgery And Clinical Research, Cianorte, v. 3, n. 22, p. 19-23, 2018. Disponível em: https://www.mastereditora.com.br/periodico/20180504 105558.pdf. Acesso em: 1 jul. 2019.

NEPA. Tabela brasileira de composição de alimentos. 4. ed. Campinas, 2009. $161 \mathrm{p}$. Disponível em: http://www.nepa.unicamp.br/taco/index.php. Acesso em: 21 set. 2018.

PAULA, M. C. et al. Processamento de bolo com a planta Pereskia aculeata Mill. (ora-pronóbis). Revista Brasileira de Produtos Agroindustriais, Campina Grande, v. 18, n. 2, p.167-174, 2016. Disponível em: http://www.deag.ufcg.edu.br/rbpa/rev182/rev1827.pdf. Acesso em: 2 fev. 2019.

PINHEIRO, D. M.; PORTO, K. R. A.; MENEZES, M. E. S. A Química dos alimentos: carboidratos, lipídeos, proteínas, vitaminas e minerais. Alagoas: EDUFAL, 2005. 52 p. Disponível em http://www.usinaciencia.ufal.br/multimidia/livros-digitais-cadernostematicos/A_Quimica_dos_Alimentos.pdf. Acesso em: 1 jul. 2019.

PINTO, N. de C. C.; SCIO, E. The biological activities and chemical composition of Pereskia Species (Cactaceae): a review. Plant Foods For Human Nutrition, Amsterdã, Netherlands, v. 69, n. 3, p. 189-195, 2014. Disponível em: https://www.ncbi.nlm.nih.gov/pubmed/24862084. Acesso em: 14 out. 2018.

QUEIROZ, C. R. A. dos A. et al. Crescimento inicial e composição química de Pereskia aculeata Miller cultivada em diferentes luminosidades. Revista Agrogeoambiental, Pouso Alegre, v. 7, n. 4, p. 93-104, 2015. Disponível em: http://dx.doi.org/10.18406/23161817v7n42015695. Acesso em: 16 jul. 2018.

ROCHA, D. R. C. et al. Macarrão adicionado de ora-pro-nobis (Pereskia aculeata Miller) desidratado. Alimentos e Nutrição, Araraquara, v. 19, n. 4, p. 459-465, 2008. Disponível em: http://serv-bib.fcfar.unesp.br/seer/index.php/alimentos/article/viewFile/656/552. Acesso em: 23 out. 218.

RODRIGUES, A. S. Atividade antioxidante e antimicrobiana de extratos de ora-pronóbis (Pereskia aculeata Mill.) e sua aplicação na mortadela. 2016. 91f. Dissertação (Mestrado em Ciência e Tecnologia em Alimentos) - Universidade Federal de Santa Maria, 
Santa Maria, 2016. Disponível em: https://repositorio.ufsm.br/handle/1/5798. Acesso em: 18 nov. 2018.

RODRIGUES, S. et al. Chemical and nutritional characterization of the ora-pro-nobis flour (Pereskia aculeata Mill.). Revista FAEF, Garça, v. 26, dez. 2014. Disponível em: http://faef.revista.inf.br/site/e/agronomia-26-edicao-dezembro-de-2014.html\#tab1142. Acesso em: 12 fev. 2019.

SANTANA, C. S. et al. Desenvolvimento de suplemento alimentar utilizando ora-pro-nóbis (Pereskia aculeata). Cadernos de Agroecologia, Recife, v.13, n. 2, p. 1-10, 2018. Disponível em: http://cadernos.aba-agroecologia.org.br/index.php/cadernos/article/view/2318. Acesso em: 19 jul. 2019.

SANTOS, A. G. et al. Estudo do efeito antimicrobiano do extrato bruto das folhas de Pereskia aculeata Mill. sobre patógenos bucais. In: MOSTRA INTERNA DE TRABALHOS DE INICIAÇÃO CIENTÍFICA CESUMAR, 5., 2010, Maringá. Anais eletrônicos[...]. Maringá: Cesumar, 2010. Disponível em:

http://www.cesumar.br/prppge/pesquisa/mostras/quin_mostra/ariane_giachini_santos_2.pdf. Acesso em: 15 fev. 2019.

SANTOS, A. G. et al. Avaliação das atividades antimicrobiana sobre patógenos bucais e hemolítica das folhas de Pereskia aculeata. In: ENCONTRO INTERNACIOANAL DE PRODUÇÃO CIENTÍFICA CESUMAR, 7., 2011, Maringá. Anais eletrônicos[...]. Maringá: Cesumar, 2011. Disponível em: https://www.unicesumar.edu.br/epcc-2011/wpcontent/uploads/sites/86/2016/07/ariane_giachini_santos.pdf. Acesso em: 10 jul. 2019.

SANTOS, L. S. et al. Análise química de folhas de cactáceas do gênero Pereskia. Agrarian, Dourados, v. 8, n. 30, p. 343-350, 2015. Disponível em: http://ojs.ufgd.edu.br/index.php/agrarian/article/view/2960/2693. Acesso em: 1 jul. 2018.

SANTOS, M. A. T. Efeito do cozimento sobre alguns fatores antinutricionais em folhas de brócolis, couve-flor e couve. Ciência e Agrotecnologia, Lavras, v. 30, p. 294-301, 2006. Disponível em: https://doi.org/10.1590/S1413-70542006000200015. Acesso em: 18 nov. 2018.

SHARIF, K. M. et al. Pharmacological relevance of primitive leafy Cactuses Pereskia. Research Journal of Biotechnology, Indore, Índia, v. 8, n.12, p. 134-142, 2013. Disponível em:

https://www.researchgate.net/publication/261722251_Pharmacological_Relevance_of_Primiti ve_Leafy_Cactuses_Pereskia. Acesso em: 10 jul. 2019.

SILVA, C. C. et al. Elaboração e aceitação de cupcakes adicionados de farinha de ora-pronóbis (Pereskia aculeata Mill) e inulina. In: JORNADA CIENTÍFICA E MOSTRA DE EXTENSÃO, 7., 2014, Bambuí. Anais eletrônicos[...]. Bambuí: IFMG: Campus Bambuí, 2014. Disponível em:

http://bambui.ifmg.edu.br/jornada_cientifica/2014/resumos/Alim/Elaboração e aceitação de cupcakes adicionados de farinha de.pdf. Acesso em: 10 jul. 2019.

SILVA, M. L. C. et al. Compostos fenólicos, carotenóides e atividade antioxidante em produtos vegetais. Semina: Ciências Agrárias, Londrina, PR, v. 3, n. 3, p. 669-682, 2010. 
Disponível em:

http://www.uel.br/revistas/uel/index.php/semagrarias/article/viewFile/6510/5926. Acesso em: 25 out. 2018.

SILVEIRA, M. G. Ensaio nutricional de Pereskia spp: hortaliça não convencional. 2015. 137 f. Tese (Doutorado em Ciência dos Alimentos) - Universidade Federal de Lavras, Lavras, 2015. Disponível em: http://repositorio.ufla.br/jspui/handle/1/10815. Acesso em: 4 dez. 2018.

SOUZA, L. F. Aspectos fitotécnicos, bromatológicos e componentes bioativos de Pereskia aculeata, Pereskia grandifolia e Anredera cordifolia. 2014. 125 f. Tese (Doutorado em Agronomia) - Universidade Federal do Rio Grande do Sul, Porto Alegre, 2014. Disponível em: https://www.lume.ufrgs.br/bitstream/handle/10183/110057/000952172.pdf?sequence=1. Acesso em: 4 dez. 2018.

SOUZA, M. C; SARTOR, C. F.; FELIPE, D. F. Comparação da ação antioxidante de uma formulação contendo extrato de Pereskia aculeata com cosméticos anti-idade presente no mercado. Revista Saúde e Pesquisa, Maringá, v. 6, n. 3, p. 461-477, 2013. Disponível em: https://periodicos.unicesumar.edu.br/index.php/saudpesq/article/view/2630. Acesso em: 9 nov. 2018.

SOUZA, M. R. de M. et al. Mineral, protein and nitrate contents in leaves of Pereskia aculeata subjected to nitrogen fertilization. Pesquisa Agropecuária Tropical, Goiânia, v. 46, n. 1, p. 43-50, 2016. Disponível em: http://www.scielo.br/pdf/pat/v46n1/1517-6398-pat-4601-0043.pdf. Acesso em: 4 dez. 2018.

TAKEITI, C. Y. et al. Nutritive evaluation of a non-conventional leafy vegetable (Pereskia aculeata Miller). International Journal of Food Sciences and Nutrition, Abingdon, UK, v. 60, n. 1, p. 148-160, 2009. Disponível em:

https://www.tandfonline.com/doi/full/10.1080/09637480802534509. Acesso em: 4 dez. 2018.

VARGAS, A. G de. Influência da sazonalidade na composição química e nas atividades antioxidante e antimicrobiana das folhas de ora-pro-nobis (Pereskia aculeata Miller). 2017. 80 f. Dissertação (Mestrado em Tecnologia de Processos Químicos e Bioquímicos) Universidade Tecnológica Federal do Paraná, Pato Branco, 2017. Disponível em: http://revistas.utfpr.edu.br/pb/index.php/SysScy/article/view/2156/1620. Acesso em: 1 mar. 2019.

ZAPPI, D.; MACHADO, N. Lista de espécies da flora do Brasil, 2010. Disponível em: http://floradobrasil.jbrj.gov.br/reflora/PrincipalUC/PrincipalUC.do;jsessionid=633126DE679 0CE1999D6E121AC920C07. Acesso em: 17 jun. 2019.

\section{DADOS DOS AUTORES:}

Lara Soares Santos

E-mail: lara.s3@hotmail.com

Curriculum Lattes: http://lattes.cnpq.br/5191298663643214 
Graduada em Tecnologia em Alimentos pelo Instituto Federal de Educação Ciência e Tecnologia do Triângulo Mineiro. Tem experiência na área de Ciência e Tecnologia de Alimentos.

\section{Carla Regina Amorim dos Anjos Queiroz}

E-mail: carlaregina@iftm.edu.br

Curriculum Lattes: http://lattes.cnpq.br/9658623177207030

Doutorado em Agronomia (Ciência do Solo) pela Universidade Estadual Paulista Júlio de Mesquita Filho, mestrado em Química, especialização em Educação e graduação em Licenciatura e Bacharelado em Química pela Universidade Federal de Uberlândia. Atualmente é professora titular do Instituto Federal de Educação Ciência e Tecnologia do Triângulo Mineiro - Campus Uberlândia. Tem experiência na área de Química, com ênfase em análise química de material vegetal, composição centesimal de alimentos e crescimento de plantas.

\section{Cláudia Maria Tomás Melo}

E-mail: claudiamelo@iftm.edu.br

Curriculum Lattes: http://lattes.cnpq.br/6936769406413477

Doutorado em Engenharia Mecânica pela Universidade Federal de Uberlândia, mestrado em Engenharia Química pela Universidade Federal de Uberlândia e graduação em Engenharia Química, também pela Universidade Federal de Uberlândia. Atualmente é professora titular e atua como docente, no Instituto Federal do Triângulo Mineiro - Campus Uberlândia, ministrando aulas para o curso de Tecnologia em alimentos, curso Técnico em Meio Ambiente, Curso de Especialização em Controle de Qualidade em processos Alimentícios e no curso de mestrado tecnológico em Ciência e Tecnologia de Alimentos. Pesquisa na área de análises físico-químicas de água, alimentos e efluentes, com base no controle de qualidade alimentício e ambiental, visando a química verde com um planeta mais sustentável. 